\title{
Eppure
}

\section{But yet}

Giovanni Lodi

Dipartimento di Scienze Biomediche, Chirurgiche e Odontoiatriche

Università degli Studi di Milano

via Beldiletto 1/3 Milano 20142

Italia

giovanni.lodi@unimi.it

Se un anno fa qualcuno ci avesse predetto di un paese costretto in casa per mesi, con mascherine per strada e code ai supermercati, camion militari che trasportano bare e aerei civili senza viaggiatori, l'avremmo preso per un pazzo o, nella migliore delle ipotesi, per un catastrofista cosmico. Eppure.

Eppure è successo. Ed è successo esattamente come era stato previsto da anni: il virus a RNA, i pipistrelli, il salto di specie, il mercato all'aperto, l'Asia. Previsto non da un singolo genio visionario, ma da molti degli scienziati che si occupano di virus influenzali. Non avevano previsto quando ma sapevano che sarebbe successo. Eppure.

Eppure non si è fatto nulla per prepararsi. Nessun paese, con pochissime eccezioni - a Taiwan solo sette morti -, aveva un piano per affrontare la pandemia. Con le conseguenze a cui stiamo assistendo.

In un articolo dello scorso giugno The Economist descriveva alcune catastrofi che potrebbero verificarsi nel futuro prossimo e alle quali non ci stiamo affatto preparando. Tra queste eruzioni vulcaniche maggiori, come quella del 1815 che cancellò un'estate e i raccolti di mezzo mondo, ed espulsioni di massa coronale. Anche queste sono eruzioni, ma della superficie solare, e si sono già verificate in un tempo pretecnologico senza grandi conseguenze. Oggi invece metterebbero in ginocchio la nostra civiltà, perché capaci di mandare in crisi reti elettriche, sistemi satellitari, internet. Le possibilità che ciò si verifichi in questo secolo? per gli ottimisti sono 50 e 50 .

I lettori più perspicaci avranno già immaginato dove voglio andare a parare. II 18 novembre sarà l'European Antibiotic Awareness Day 2020, ovvero il giorno dedicato alla lotta alle resistenze batteriche, fenomeno che è da anni in crescita costante e che nel volgere di pochi lustri potrebbe diventare una causa di morte più frequente del cancro.

Ma mentre su sole e vulcani non abbiamo alcun controllo, le resistenze batteriche le stiamo causando noi, con i nostri comportamenti. Noi medici, noi pazienti, noi genitori, noi insegnanti, noi aziende, noi politici. Eppure.

Buona lettura, 